\title{
Association between clinicopathological features of breast cancer with adipocytokine levels and oxidative stress markers before and after chemotherapy
}

\author{
JOANNA GRUPIŃSKA ${ }^{1,2}$, MAGDALENA BUDZYŃ $^{1}$, JACEK J. BRZEZIŃSKI $^{3}$, BOGNA GRYSZCZYŃSKA ${ }^{1}$, \\ MAGDALENA P. KASPRZAK ${ }^{1}$, WITOLD KYCLER ${ }^{3}$, EWA LEPOROWSKA ${ }^{4}$ and MARIA ISKRA ${ }^{1}$ \\ ${ }^{1}$ Chair and Department of Medical Chemistry and Laboratory Medicine, Poznan University of Medical Sciences, \\ 60-806 Poznań; ${ }^{2}$ Nutrition Laboratory, Hospital Pharmacy, ${ }^{3}$ Gastrointestinal Surgical Oncology Department, \\ ${ }^{4}$ Department of Laboratory Diagnostics, Greater Poland Cancer Centre, 61-866 Poznań, Poland
}

Received August 19, 2020; Accepted December 22, 2020

DOI: 10.3892/br.2021.1406

\begin{abstract}
Adipocytokines and markers of oxidative stress have been shown to exhibit potential for detection of advanced stage, HER 2/neu status and lymph node metastases in patients with breast cancer, as well as in determining the efficiency of anti-cancer treatments. In the present study, blood concentrations of apelin (APLN), retinol-binding protein 4 (RBP4), 8-hydroxydeoxyguanosine (8-oxo-dG) and total antioxidant capacity (TAC) in women with breast cancer with different clinicopathological features were measured prior to and following adjuvant chemotherapy. The study included 60 women with breast cancer stratified according to tumor grade and size, HER-2/neu expression, and lymph node and hormone receptor status. Blood samples were taken before and after two cycles of adjuvant chemotherapy. None of the clinicopathological features were associated with the baseline concentrations of RBP4, 8-oxo-dG or TAC. An increased baseline concentration of APLN was observed in HER-2/neu positive patients. Moreover, through multivariate logistical regression analysis, APLN was shown to be independently associated with a positive HER/neu status. Chemotherapy treatment did not affect the levels of RBP4 or APLN, or TAC values when assessing all the patients, and when assessing the stratified groups of patients. Only 8-oxo-dG was found to be significantly decreased following drug administration $(\mathrm{P}=0.0009)$. This preliminary study demonstrated that APLN is a significant and independent predictor of HER-2/neu positive breast cancer. A significant reduction in 8-oxo-dG levels
\end{abstract}

Correspondence to: Dr Joanna Grupińska, Chair and Department of Medical Chemistry and Laboratory Medicine, Poznan University of Medical Sciences, 8 Rokietnicka, 60-806 Poznań, Poland E-mail: jgrupinska@ump.edu.pl

Key words: breast cancer, apelin, retinol-binding protein 4, 8-hydroxydeoxyguanosine, adipocytokines, chemotherapy, total antioxidant capacity, HER-2/neu following chemotherapy may indicate its potential clinical utility in monitoring the effects of chemotherapy in breast cancer patients.

\section{Introduction}

Breast cancer is the most common malignancy and leading cause of cancer-associated death in women between the ages of 35 and 50. Obesity is a well-established risk factor for development of breast cancer, exerting its effect via several different biological mechanisms $(1,2)$. As an endocrine organ, adipose tissue secretes molecules called adipocytokines that act in an endocrine, paracrine and autocrine manner, and may promote the malignant progression of breast cancer (3). Several studies have indicated that adipocytokines mediate the survival, growth, invasion and metastasis of breast cancer cells (4-7). Apelin (APLN) and retinol-binding protein 4 (RBP4) are adipocytokines that may serve a role in carcinogenesis (8-10). Evaluation of their role may be useful in predicting survival times and cancer recurrence (11-13).

APLN was isolated as an endogenous ligand from bovine stomach epithelial cells in 1998 (14). Further studies have demonstrated that APLN is also expressed in heart muscles, brain, kidneys, liver, lungs and spleen, as well as in mammary glands, placenta and gastric mucosa (15). The small peptide is involved in several vital physiological processes, such as angiogenesis, fluid homeostasis and glucose metabolism (16). Growing evidence has suggested that APLN induces the maturation of tumor blood capillaries and prompts tumor vascularization (17). Moreover, APLN also shows lymph angiogenic potential in relation to tumor growth and lymph node metastasis (18). Upregulated expression of APLN has been found in various types of cancer, including breast cancer, where its levels have been shown to be correlated with shorter survival times and a higher incidence of cancer recurrence (19-21).

RBP4 is a more recently identified adipokine that transports retinol (vitamin A) from the liver to peripheral tissues (11). In adipose tissues, RBP4 is expressed in mature, lipid-laden adipocytes (22). It has been observed that increased RBP4 
is positively correlated with obesity-linked complications, including impaired glucose tolerance, insulin resistance, type 2 diabetes mellitus, dyslipidemia, hypertension and cardiovascular disease (23). Studies have also indicated that upregulated expression of RBP4 is associated with colorectal, ovarian and endometrial cancer (24-26). Recently Jiao et al (11) reported that elevated RBP4 concentrations were associated with an increased risk of breast cancer independent of BMI, lipid levels and other potential risk factors (11). The specific role of RBP4 in carcinogenesis is not understood, to the best of our knowledge. It has been shown to potentiate migration and proliferation of tumor cells by stimulating the synthesis of MMP-2 and MMP-9, thus facilitating tumor cell infiltration in surrounding tissues (25).

It is well documented that carcinogenesis is associated with the overproduction of reactive oxygen species (ROS) (27-29). ROS can cause oxidative damage to lipids, proteins and DNA. Oxidized and damaged DNA (resulting in genetic mutations) is involved in malignant transformation. 8-hydroxydeoxyguanosine (8-oxo-dG) is a specific marker of 2-deoxyguanosine damage following ROS-mediated damage to DNA. High levels of 8-oxo-dG in tumors, blood samples or urine have been found in patients with various types of cancer $(30,31)$. Moreover, 8-oxo-dG may be useful for predicting prognosis in different types of cancer $(32,33)$. ROS levels are maintained within narrow limits under physiological conditions by an antioxidant defense system consisting of multiple independent components (34). However, there is evidence of impaired antioxidant status in patients with various types of cancer, including breast cancer, due to an imbalance between ROS production and elimination, resulting in oxidative damage to key biomolecules (35). Furthermore, recent studies have indicated a close correlation between adipocytokines and oxidative stress, where certain adipocytokines were shown to increase the production of free radicals, whereas others inhibited this process $(31,36-38)$.

Although alterations in the levels of adipocytokines and markers of oxidative stress in patients with breast cancer are well documented, relatively little is known regarding the degree to which they vary in different breast cancer subtypes or in relation to the aggressiveness of the breast cancer $(39,40)$. Breast cancer is a complex and heterogeneous disease, and its prognosis may vary based on the combined characteristics of the tumor itself and any underlying conditions a patient may have (41). The role of obesity-related markers and oxidative stress in tumor growth and metastasis may be associated with distinct subtypes of breast cancer. Therefore, in the present study, the concentrations of APLN, RBP4 and 8-oxo-dG in breast cancer patients with different clinicopathological features, including tumor grade and size, HER-2/neu expression, hormone receptor status and lymph node status were investigated. Due to the fact that there is a great interest in identifying biomarkers that may be used to predict chemotherapy treatment responses, the second aim of the present study was to analyze the effects of 6-week adjuvant chemotherapy on APLN, RBP4 and 8-oxo-dG levels.

\section{Materials and methods}

Patients and methods. The study cohort was formed of women with breast cancer treated at the Greater Poland Cancer Center in Poznan between December 2016 to November 2017 who qualified for adjuvant chemotherapy. The study was performed according to principles of the Declaration of Helsinki (42). The protocol used in the present study was approved by the Local Bioethical Committee of Poznan University of Medical Sciences (approval no. 1016/16). The study included 60 women and written informed consent was obtained from all participants. The median age was 58 years old (range, 31-76). Selected clinical criteria collected in the pre-operative period included tumor histological grade and size, HER-2/neu expression, and the presence/absence of regional lymph node metastases, and this data was used to divide patients into different categories. The histological grading of breast tumors was based on the Modified Bloom-Richardson Grading Scheme (43). The status the HER2-neu, progesterone and estrogen receptor expression was assessed by immunohistochemistry (IHC) analysis at the Department of Cancer Pathology, Greater Poland Cancer Center, as a routine diagnostic procedure, using the EnVision ${ }^{\mathrm{TM}}+\mathrm{HRP}$ complex (DakoCytomation; Agilent Technologies, Inc.). HER2 status (HercepTest ${ }^{\mathrm{TM}}$; Agilent Technologies, Inc.) was scored as follows: 0 , no staining; 1 , weakly visible staining in $>10 \%$ of neoplastic cells; 2 , weak or moderate staining in $>10 \%$ of neoplastic cells; and 3, strong staining in $>10 \%$ of neoplastic cells. In the case of moderate expression (2+), a FISH test was performed to assess HER2-neu gene amplification using HER2 IQFISH pharmDx ${ }^{\mathrm{TM}}$ kit (Dako Omnis; Agilent Technologies, Inc.). HER2 IQFISH pharmDx ${ }^{\mathrm{TM}}$ reagent (Dako Omnis; Agilent Technologies, Inc.) was used as a reference standard for HER2. Assessment of HER2 gene amplification with the HER2 IQFISH pharmDx ${ }^{\mathrm{TM}}$ test (Dako Omnis) was fully automated and was performed on the Dako Omnis device according tot the manufacturer's protocol. For FISH analysis, the slides were deparaffinized by immersing them in Clearify ${ }^{\mathrm{TM}}$ for $10 \mathrm{~min}$, unmasking the antigen using ISH Pre-Treatment Solution (Dako Omnis; Agilent Technologies, Inc.) for $15 \mathrm{~min}$, followed by dehydration in $96 \%$ ISH Ethanol Solution (Dako Omnis; Agilent Technologies, Inc.) at $32^{\circ} \mathrm{C}$ twice for $3 \mathrm{~min}$. Subsequently, slides were treated with pepsin for $15 \mathrm{~min}$ and finally air dried at $45^{\circ} \mathrm{C}$ for $15 \mathrm{~min}$. Next, the slides were subjected to denaturation by immersing them in a denaturing solution at $66^{\circ} \mathrm{C}$ for $10 \mathrm{~min}$. Slides underwent hybridization by applying HER2 IQFISH pharmDx ${ }^{\mathrm{TM}}$ (Dako Omnis; Agilent Technologies, Inc.). HER2 IQFISH pharmDx ${ }^{\mathrm{TM}}$ reagent (Dako Omnis; Agilent Technologies, Inc.) is a mix of IQISH probes consisting of a mix of Texas red labeled DNA probes $(218 \mathrm{~kb}$ long region) containing the HER2 gene on chromosome 17, and a mix of fluorescein labeled probes peptide nucleic acid directed against the centromeric region of chromosome 17 (CEN-17). Specific hybridization with target regions results in a distinct red fluorescent signal for each HER2 gene locus and a green fluorescent signal for each centromere of chromosome 17. The slides were hybridized at $45^{\circ} \mathrm{C}$ for $75 \mathrm{~min}$. A deep rinse was performed with ISH Stringent Wash Buffer (Dako Omnis ${ }^{\mathrm{TM}}$; Agilent Technologies, Inc.) at $61^{\circ} \mathrm{C}$ for $10 \mathrm{~min}$. After staining in the Dako Omnis machine, sections were mounted on a slide with Fluorescence Mounting Medium (Dako Omnis ${ }^{\mathrm{TM}}$; Agilent Technologies, Inc.) containing DAPI and then covered with a coverslip. Using a fluorescence microscope equipped with appropriate filters, the position of tumor cells was determined and the red (HER2) and green (CEN-17) signals were counted, 
and the HER2/CEN-17 ratio was calculated. Healthy cells in the analyzed tissue sections served as the internal control for positive staining. IHC scores of $3+$ and $2+$ with positive HER2 amplification was considered to be indicative of positive HER2-neu receptor activity. IHC $0,1+$ and $2+$ with negative HER2 amplification was taken to be negative HER2-neu receptor activity. To assess the activity of estrogen and progesterone receptors, monoclonal antibodies against estrogen (cat. no. 1D5; 1:50; DakoCytomation; Agilent Technologies, Inc.) and progesterone receptors (cat. no. PgR636; 1:50; DakoCytomation; Agilent Technologies, Inc.) and a polyclonal antibody against estrogen $\beta$ receptors (Chemicon) were used. The presence of estrogen and/or progesterone receptors on the tumor surface defined the tumor as hormone dependent. The characteristics of the study group are presented in Table I. All patients underwent four cycles of adjuvant chemotherapy in a regimen involving doxorubicin and cyclophosphamide (AC). HER2-positive patients received trastuzumab in addition to $\mathrm{AC}$.

Blood samples for biochemical analyses were taken from the antecubital vein 1 day prior to chemotherapy after an overnight fast, and 6 weeks later when the first and second cycles of chemotherapy were completed. Samples were collected in EDTA anticoagulant serum tubes. After $30 \mathrm{~min}$, the tubes were centrifuged at $1,000 \mathrm{x}$ g for $15 \mathrm{~min}$. Serum and plasma samples were stored at a temperature of $-80^{\circ} \mathrm{C}$ until required for assay. The concentrations of RBP4, APLN and 8-oxo-dG were measured using ELISA kits according to the manufacturer's protocol (AssayPro LLC, cat. no. ER3005-1; Phoenix Pharmaceuticals, cat. no. EKE-057-15, Inc, EIAab, cat. no. E0660Ge). Total antioxidant capacity (TAC) was determined using an Antioxidant assay kit (Cayman Chemical Company) and expressed in Trolox equivalents. Trolox is a water-soluble analog of tocopherol used to assess the antioxidant potential of a mixture containing several antioxidants.

Women with diabetes mellitus, cardiovascular diseases, hypertension, and inflammatory diseases were not included in the present study.

Statistical analysis. Statistical analysis was performed using Statistica version 12.0 (StatSoft Inc.). The normality of quantitative variables was assessed using a Kolmogorov-Smirnov or Shapiro-Wilk test. Normally distributed, continuous variables were presented as the mean \pm standard deviation. Parameters that were not normally distributed are presented as a median (range). Comparisons between appropriate categories of patients were made using an unpaired Student's t-test or a Mann-Whitney U test depending on the distribution of the data. A comparison between analyzed parameters before and after chemotherapy was performed using a paired Student's t-test or a Wilcoxon test, depending on the distribution of the data. Pearson's or Spearman's correlation coefficient analysis was used to assess the strength of any association between different variables. Multivariate logistical regression analysis was performed using APLN as an independent variable and HER-2/neu status as a dependent variable after adjusting for age, BMI, adipose tissue content, TAC value, 8-oxo-dG levels, lymph node status, hormone receptor status, tumor grade and size. $\mathrm{P}<0.05$ was considered to indicate a statistically significant difference.
Table I. Clinicopathological characteristics of the study group.

\begin{tabular}{lc}
\hline Clinicopathological feature & $\mathrm{N}(\%)$ \\
\hline Histopathological grade & $31(51.7)$ \\
I/II & $29(48.3)$ \\
III & \\
HER-2/neu expression & $20(33.3)$ \\
+ & $40(66.7)$ \\
- & \\
Tumor size & $33(55)$ \\
$<2$ cm & $27(45)$ \\
$>2$ cm & \\
Regional lymph node metastases & $32(53.3)$ \\
Present & $28(46.7)$ \\
Absent & \\
Hormonal sensitivity & $44(73.3)$ \\
Hormonal-positive & $16(26.7)$ \\
Hormonal-negative &
\end{tabular}

\section{Results}

Baseline concentrations of adipocytokines and markers of oxidative stress in patients with breast cancer. First, patients were divided into subgroups according to selected clinical features, including histological tumor grade and size, HER-2/neu expression, hormone receptor status, the presence/absence of metastases in regional lymph nodes. The concentrations of APLN, RBP4 and 8-oxo-dG, as well as the TAC levels were assessed. All parameters were evaluated before administration of any chemotherapy.

No differences in adipose tissue content and BMI values were observed between the groups. RBP4 and 8-oxo-dG levels also did not differ between the groups. RBP4 and 8-oxo-dG concentrations did not differ amongst patients based on tumor size, tumor grade, HER-2/neu status, presence of lymph node metastases or hormone receptor status (Table II). Only APLN levels were found to be significantly higher in HER-2/neu positive cases compared with HER-2/neu negative patients (Table IIC). In addition, only TAC levels were significantly higher in women with hormone-positive breast cancer (Table IIE).

Logistical regression showed that higher APLN levels were significantly associated with positive HER-2/neu status (Table III, Model 1), even after adjusting for potential confounding factors, such as age, BMI, adipose tissue content, markers of oxidative stress (Table III, Model 2), tumor grade and size, hormone receptor status and lymph node metastasis (Table III, Model 3).

Univariate analysis performed on the levels before chemotherapy demonstrated that neither APLN nor RBP4 concentrations were correlated with patient age, BMI or the parameters of oxidative stress (Table IV). RBP4 concentrations were only positively associated with adipose tissue content (Table IV). 8-oxo-dG levels were not correlated with age, adipose tissue content or TAC. However, there was a 
Table II. Concentration of adipocytokines and selected markers of oxidative stress in women with breast cancer.

A, Tumor size

\begin{tabular}{lccc}
\hline Analyzed parameter & $<2 \mathrm{~cm}$ & $>2 \mathrm{~cm}$ & P-value \\
\hline $\mathrm{RBP} 4, \mu \mathrm{g} / \mathrm{ml}$ & $67.14(55.07-103.7)$ & $69.02(42.85-105.4)$ & 0.741 \\
$\mathrm{APLN}, \mathrm{ng} / \mathrm{ml}$ & $1.31 \pm 0.39$ & $1.26 \pm 0.47$ & 0.721 \\
$8-\mathrm{oxo}-\mathrm{dG}, \mathrm{ng} / \mathrm{ml}$ & $10.1(5.80-26.54)$ & $3.32(2.02-9.20)$ & 0.886 \\
$\mathrm{TAC}, \mu \mathrm{g} / \mathrm{ml}$ & $2.85(0.62-12.13)$ & $27.2(17.70-37.40)$ & 0.156 \\
$\mathrm{BMI}, \mathrm{kg} / \mathrm{m}^{2}$ & $25.7(19.70-41.80)$ & $31.8(15.30-43.40)$ & 0.950 \\
Adipose tissue content, $\%$ & $33.5(18.80-44.30)$ & $57.48 \pm 10.71$ & 0.223 \\
Age & $58.36 \pm 9.30$ & & 0.734 \\
\hline
\end{tabular}

B, Histopathological grade

\begin{tabular}{lccc}
\hline Analyzed parameter & I/II & III & P-value \\
\hline $\mathrm{RBP} 4, \mu \mathrm{g} / \mathrm{ml}$ & $66.96(55.07-103.4)$ & $69.02(42.85-105.4)$ & 0.457 \\
$\mathrm{APLN}, \mathrm{ng} / \mathrm{ml}$ & $1.25 \pm 0.40$ & $1.33 \pm 0.45$ & 0.558 \\
$8-\mathrm{oxo}-\mathrm{dG}, \mathrm{ng} / \mathrm{ml}$ & $9.28(5.80-30.94)$ & $2.91(0.62-9.20)$ & 0.392 \\
$\mathrm{TAC}, \mu \mathrm{g} / \mathrm{ml}$ & $2.98(1.26-12.13)$ & $26.8(17.70-41.80)$ & 0.968 \\
$\mathrm{BMI}, \mathrm{kg} / \mathrm{m}^{2}$ & $25.3(18.30-36.50)$ & $35.4(18.50-44.30)$ & 0.142 \\
Adipose tissue content, $\%$ & $31.6(15.30-43.0)$ & $57.38 \pm 11.14$ & 0.095 \\
Age & $58.52 \pm 8.69$ & & 0.659 \\
\hline
\end{tabular}

C, HER-2/neu expression

\begin{tabular}{lccc}
\hline Analyzed parameter & + & - & P-value \\
\hline $\mathrm{RBP} 4, \mu \mathrm{g} / \mathrm{ml}$ & $79.41(42.85-103.7)$ & $67.14(55.38-105.4)$ & 0.780 \\
$\mathrm{APLN}, \mathrm{ng} / \mathrm{ml}$ & $1.58 \pm 0.37$ & $1.16 \pm 1.00$ & $0.002^{\mathrm{b}}$ \\
$8-\mathrm{oxo}-\mathrm{dG}, \mathrm{ng} / \mathrm{ml}$ & $9.96(4.99-31.61)$ & $2.91(1.26-9.20)$ & 0.893 \\
$\mathrm{TAC}, \mu \mathrm{g} / \mathrm{ml}$ & $3.57(0.62-12.13)$ & $27(17.70-37.40)$ & 0.408 \\
$\mathrm{BMI}, \mathrm{kg} / \mathrm{m}^{2}$ & $25.2(19.70-41.80)$ & $33.4(15.30-44.30)$ & 0.541 \\
Adipose tissue content, $\%$ & $32(18.80-44.00)$ & $57.20 \pm 10.37$ & 0.632 \\
Age & $59.50 \pm 8.86$ & & 0.399 \\
\hline
\end{tabular}

D, Regional lymph node metastases

\begin{tabular}{lccc}
\hline Analyzed parameter & Present & Absent & P-value \\
\hline $\mathrm{RBP} 4, \mu \mathrm{g} / \mathrm{ml}$ & $66.21(42.85-105.4)$ & $72.92(55.07-103.7)$ & 0.268 \\
$\mathrm{APLN}, \mathrm{ng} / \mathrm{ml}$ & $1.24 \pm 0.47$ & $1.33 \pm 0.37$ & 0.479 \\
$8-\mathrm{oxo}-\mathrm{dG}, \mathrm{ng} / \mathrm{ml}$ & $10.15(4.51-30.94)$ & $9.05(3.68-31.61)$ & 0.545 \\
$\mathrm{TAC}, \mu \mathrm{g} / \mathrm{ml}$ & $3.48(1.26-12.13)$ & $2.72(0.62-9.80)$ & 0.321 \\
$\mathrm{BMI}, \mathrm{kg} / \mathrm{m}^{2}$ & $25.5(17.70-37.40)$ & $26.2(21.20-41.80)$ & 0.51 \\
Adipose tissue content, $\%$ & $31.8(15.30-43.40)$ & $33.55(21.90-44.30)$ & 0.219 \\
Age & $58.75 \pm 10.14$ & $57.07 \pm 9.68$ & 0.516 \\
\hline
\end{tabular}

E, Hormone receptor status

\begin{tabular}{lccc}
\hline Analyzed parameter & Positive & Negative & P-value \\
\hline RBP4,$\mu \mathrm{g} / \mathrm{ml}$ & $66.95(42.85-105.4)$ & $72.92(57.61-103.4)$ & 0.277 \\
APLN, ng/ml & $1.22 \pm 1.07$ & $0.87 \pm 0.55$ & 0.265 \\
$8-\mathrm{oxO}-\mathrm{dG}, \mathrm{ng} / \mathrm{ml}$ & $8.65(3.68-30.94)$ & $14.07(5.80-31.61)$ & 0.086
\end{tabular}


Table II. Continued.

E, Hormone receptor status

\begin{tabular}{lccc}
\hline Analyzed parameter & Positive & Negative & P-value \\
\hline TAC,$\mu \mathrm{g} / \mathrm{ml}$ & $3.18(0.62-12.13)$ & $2.07(1.26-5.99)$ & $0.043^{\mathrm{a}}$ \\
$\mathrm{BMI}, \mathrm{kg} / \mathrm{m}^{2}$ & $26.6(17.70-37.40)$ & $24.85(19.50-41.80)$ & 0.640 \\
Adipose tissue content, $\%$ & $32.95(15.30-44.30)$ & $33.25(19.50-44.00)$ & 0.676 \\
Age & $57.8 \pm 8.83$ & $58.44 \pm 12.66$ & 0.826 \\
\hline
\end{tabular}

${ }^{\mathrm{a}} \mathrm{P}, 0.05,{ }^{\mathrm{b}} \mathrm{P}<0.01$. RBP4, retinol-binding protein 4; APLN, apelin; 8-oxo-dG, 8-hydroxydeoxyguanosine; TAC, total antioxidant capacity.

Table III. Multivariate logistical regression analysis of the association between APLN and HER-2/neu status .

\begin{tabular}{lccc}
\hline Variable & Odds ratio & $95 \%$ confidence interval & P-value \\
\hline Model 1 & 2.14 & $0.483-10.73$ & $0.038^{\mathrm{a}}$ \\
Model 2 & 26.84 & $1.627-1363$ & $0.045^{\mathrm{a}}$ \\
Model 3 & 91.15 & $3.142-30162$ & $0.043^{\mathrm{a}}$ \\
\hline
\end{tabular}

ap $<0.05$. Model 1, unadjusted APLN; Model 2, adjusted for age, BMI, adipose tissue content, 8-oxo-dG, TAC value; Model 3, adjusted for tumor size, histopathological grade, lymph node status and hormone receptor status; APLN, apelin.

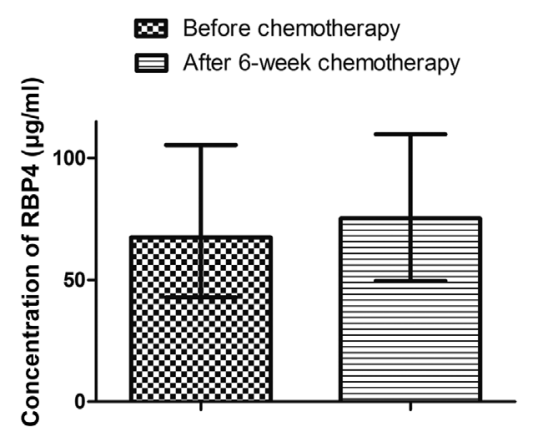

Figure 1. Difference in RBP4 levels after 6-weeks of chemotherapy. Data were analyzed using a Wilcoxon test. RBP4, retinol-binding protein 4 .

significant negative association between 8 -oxo-dG levels and BMI (Table IV).

Concentration of adipocytokines and markers of oxidative stress after 6-weeks of chemotherapy. The effects of 6-week chemotherapy on APLN, RBP4, 8-oxo-dG and TAC levels in the entire group, as well as within the different subgroups was next addressed.

Levels of RBP4 in the total group of women increased from an initial value of $67.51 \mu \mathrm{g} / \mathrm{ml}(42.85-105.4 \mu \mathrm{g} / \mathrm{ml})$ to $75.27 \mu \mathrm{g} / \mathrm{ml}(49.56-109.8 \mu \mathrm{g} / \mathrm{ml})$ after 6-weeks of chemotherapy (Fig. 1). However, this increase was not statistically significant $(\mathrm{P}=0.2066)$.

No change in RBP4 concentration were observed after 6-weeks of chemotherapy in any of the sub-categories based

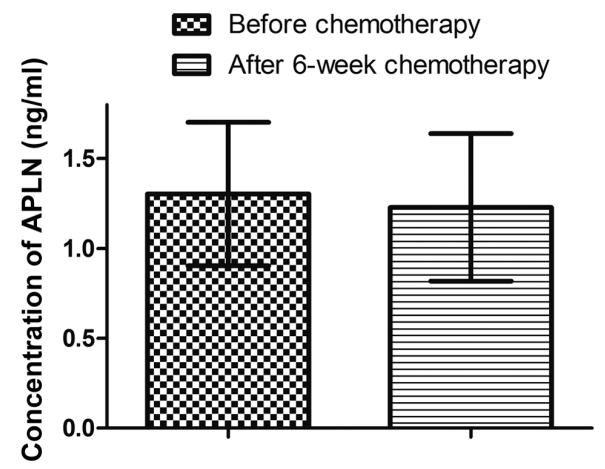

Figure 2. APLN concentration before and after 6-weeks of chemotherapy. Data were analyzed using a paired Student's t-test. APLN, apelin.

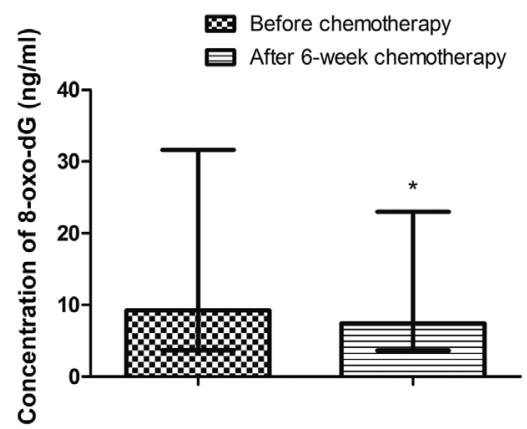

Figure 3. Concentration of 8-oxo-dG before and after 6-weeks of chemotherapy. Data were analyzed using a Wilcoxon test. ${ }^{*} \mathrm{P} \leq 0.05 .8$-oxo-dG, 8-hydroxydeoxyguanosine.

on tumor histological grade and size, HER-2/neu expression, hormone receptor status, and the presence/absence of regional lymph node metastases (Table V).

The concentrations of APLN prior to chemotherapy and 6 weeks after were $1.30 \pm 0.40$ and $1.23 \pm 0.41 \mathrm{ng} / \mathrm{ml}$, respectively. However, this reduction was not significantly different ( $\mathrm{P}=0.2058$; Fig. 2).

APLN concentration was not significantly altered in any of the subgroups after 6-weeks of chemotherapy (Table VI).

The concentration of 8-oxo-dG decreased significantly when comparing all the data together, from $9.23 \mathrm{ng} / \mathrm{ml}$ (3.68-31.61 ng/ml) to $7.42 \mathrm{ng} / \mathrm{ml}(3.64-22.97 \mathrm{ng} / \mathrm{ml})$ after 6-weeks of chemotherapy; $\mathrm{P}=0.0009$ (Fig. 3). A similar observation was seen when comparing patients with tumors $>2 \mathrm{~cm}(\mathrm{P}=0.007)$, with 
Table IV. Univariate analysis for association between parameters before and after treatment.

A, Before chemotherapy

\begin{tabular}{|c|c|c|c|c|c|c|}
\hline \multirow[b]{2}{*}{ Correlated parameter } & \multicolumn{2}{|c|}{ APLN } & \multicolumn{2}{|c|}{ RBP4 } & \multicolumn{2}{|c|}{ 8-oxo-dG } \\
\hline & $\mathrm{r}$ & P-value & $\mathrm{r}$ & $\mathrm{P}$-value & $\mathrm{r}$ & $\mathrm{P}$-value \\
\hline Age & 0.057 & $0.728^{\mathrm{b}}$ & 0.118 & $0.456^{\mathrm{c}}$ & 0.030 & $0.864^{\mathrm{c}}$ \\
\hline BMI & -0.084 & $0.613^{\mathrm{b}}$ & 0.271 & $0.082^{c}$ & -0.343 & $0.040^{\mathrm{a}, \mathrm{c}}$ \\
\hline Adipose tissue content & -0.237 & $0.147^{\mathrm{b}}$ & 0.343 & $0.026^{\mathrm{a}, \mathrm{c}}$ & -0.225 & $0.186^{\mathrm{c}}$ \\
\hline TAC & -0.18 & $0.293^{c}$ & -0.005 & $0.974^{\mathrm{c}}$ & 0.204 & $0.232^{\mathrm{c}}$ \\
\hline 8-oxo-dG & -0.01 & $0.955^{\mathrm{c}}$ & 0.004 & $0.981^{\mathrm{c}}$ & - & - \\
\hline
\end{tabular}

B, After 6-weeks of chemotherapy

\begin{tabular}{|c|c|c|c|c|c|c|}
\hline \multirow[b]{2}{*}{ Correlated parameter } & \multicolumn{2}{|c|}{ APLN } & \multicolumn{2}{|c|}{ RBP4 } & \multicolumn{2}{|c|}{ 8-oxo-dG } \\
\hline & $\mathrm{r}$ & P-value & $\mathrm{r}$ & $\mathrm{P}$-value & $\mathrm{r}$ & P-value \\
\hline Age & -0.028 & $0.868^{\mathrm{b}}$ & 0.162 & $0.305^{\mathrm{b}}$ & 0.039 & $0.822^{\mathrm{c}}$ \\
\hline BMI & 0.104 & $0.530^{\mathrm{b}}$ & 0.230 & $0.143^{b}$ & -0.262 & $0.123^{c}$ \\
\hline Adipose tissue content & 0.295 & $0.069^{\mathrm{b}}$ & 0.318 & $0.040^{\mathrm{a}, \mathrm{b}}$ & -0.202 & $0.236^{c}$ \\
\hline TAC & 0.161 & $0.348^{\mathrm{c}}$ & -0.160 & $0.317^{\mathrm{c}}$ & 0.365 & $0.029^{\mathrm{a}, \mathrm{c}}$ \\
\hline 8-oxo-dG & -0.240 & $0.194^{\mathrm{c}}$ & -0.096 & $0.579^{c}$ & - & - \\
\hline
\end{tabular}

${ }^{\mathrm{a}} \mathrm{P}<0.05$. ${ }^{\mathrm{b}} \mathrm{Pearson}$ correlation coefficient; ${ }^{\mathrm{c}}$ Spearman correlation coefficient.

Table V. Effect of 6-weeks of chemotherapy on retinol-binding protein 4 concentration in women with breast cancer.

\begin{tabular}{|c|c|c|c|}
\hline Clinicopathological feature & Before chemotherapy, $\mu \mathrm{g} / \mathrm{ml}^{\mathrm{a}}$ & After 6-weeks of chemotherapy, $\mu \mathrm{g} / \mathrm{ml}^{\mathrm{a}}$ & P-value \\
\hline \multicolumn{4}{|l|}{ Histopathological grade } \\
\hline $\mathrm{I} / \mathrm{II}$ & $66.96(55.07-103.4)$ & $72.03(49.56-99.82)$ & 0.537 \\
\hline III & $69.02(42.85-105.4)$ & $80.48(53.68-105.4)$ & 0.254 \\
\hline \multicolumn{4}{|l|}{ HER-2/neu expression } \\
\hline+ & $79.41(42.85-103.7)$ & $79.82(49.56-109.8)$ & 0.791 \\
\hline- & $67.14(55.38-105.4)$ & $73.63(56.48-102.6)$ & 0.174 \\
\hline \multicolumn{4}{|l|}{ Tumor size } \\
\hline$<2 \mathrm{~cm}$ & $67.14(55.07-103.7)$ & $72.93(49.56-109.8)$ & 0.558 \\
\hline$>2 \mathrm{~cm}$ & $69.02(42.85-105.4)$ & $77.78(53.68-99.82)$ & 0.239 \\
\hline \multicolumn{4}{|c|}{ Regional lymph node metastases } \\
\hline Present & $66.21(42.85-105.4)$ & $67.69(53.68-99.82)$ & 0.424 \\
\hline Absent & $72.92(55.07-103.7)$ & $81.58(49.56-109.8)$ & 0.348 \\
\hline \multicolumn{4}{|l|}{ Hormonal sensitivity } \\
\hline Hormonal-positive & $66.95(42.85-105.4)$ & $76.4(49.56-99.82)$ & 0.213 \\
\hline Hormonal-negative & $72.92(57.61-103.4)$ & $70.94(56.48-109.8)$ & 0.7 \\
\hline
\end{tabular}

${ }^{\mathrm{a}}$ Median (range).

metastases in the regional lymph nodes $(\mathrm{P}=0.004)$, irrespective of the HER-2/neu status (HER2/neu positive $\mathrm{P}=0.021$; HER2/neu negative $\mathrm{P}=0.017$ ) (Table VII). A decrease in 8-oxo-dG levels was observed regardless of tumor grade ( $\mathrm{I} / \mathrm{II} \mathrm{P}=0.007$; III $\mathrm{P}=0.041$ ) and hormone receptor status (hormone-positive $\mathrm{P}=0.038$; hormone-negative $\mathrm{P}=0.004$ ) (Table VII).

TAC values increased after 6 weeks of chemotherapy in the entire group of women from an initial value of $2.97 \mathrm{mmol} / \mathrm{l}$ 
Table VI. Effect of 6-weeks of chemotherapy on apelin concentration in women with breast cancer.

\begin{tabular}{|c|c|c|c|}
\hline Clinicopathological feature & Before chemotherapy, ng/ml ${ }^{\mathrm{a}}$ & After 6-weeks of chemotherapy, $\mathrm{ng} / \mathrm{ml}^{\mathrm{a}}$ & P-value \\
\hline \multicolumn{4}{|l|}{ Histopathological grade } \\
\hline $\mathrm{I} / \mathrm{II}$ & $1.25 \pm 0.40$ & $1.21 \pm 0.47$ & 0.661 \\
\hline III & $1.33 \pm 0.45$ & $1.23 \pm 0.38$ & 0.223 \\
\hline \multicolumn{4}{|l|}{ HER-2/neu expression } \\
\hline+ & $1.58 \pm 0.37$ & $1.46 \pm 0.44$ & 0.415 \\
\hline- & $1.16 \pm 1.00$ & $1.11 \pm 0.38$ & 0.459 \\
\hline \multicolumn{4}{|l|}{ Tumor size } \\
\hline$<2 \mathrm{~cm}$ & $1.31 \pm 0.39$ & $1.26 \pm 0.43$ & 0.561 \\
\hline$>2 \mathrm{~cm}$ & $1.26 \pm 0.47$ & $1.17 \pm 0.43$ & 0.306 \\
\hline \multicolumn{4}{|c|}{ Regional lymph node metastases } \\
\hline Present & $1.24 \pm 0.47$ & $1.11 \pm 0.40$ & 0.101 \\
\hline Absent & $1.33 \pm 0.37$ & $1.33 \pm 0.42$ & 0.937 \\
\hline \multicolumn{4}{|l|}{ Hormonal sensitivity } \\
\hline Hormonal-positive & $1.22 \pm 1.07$ & $1.08 \pm 0.55$ & 0.290 \\
\hline Hormonal-negative & $0.87 \pm 0.55$ & $0.74 \pm 0.42$ & 0.095 \\
\hline
\end{tabular}

${ }^{\mathrm{a}}$ Mean \pm standard deviation.

Table VII. Effect of 6-weeks of chemotherapy on 8-hydroxydeoxyguanosine concentration in women with breast cancer.

\begin{tabular}{|c|c|c|c|}
\hline Clinicopathological feature & Before chemotherapy, ng/ml ${ }^{\mathrm{c}}$ & After 6-weeks of chemotherapy, $\mathrm{ng} / \mathrm{ml}^{\mathrm{c}}$ & P-value \\
\hline \multicolumn{4}{|l|}{ Histopathological grade } \\
\hline $\mathrm{I} / \mathrm{II}$ & $9.28(5.80-30.94)$ & $7.26(3.90-22.97)$ & $0.007^{\mathrm{b}}$ \\
\hline III & $9.19(3.68-31.61)$ & $7.44(3.64-14.68)$ & $0.041^{\mathrm{a}}$ \\
\hline \multicolumn{4}{|l|}{ HER-2/neu expression } \\
\hline+ & $9.96(4.99-31.61)$ & $7.5(3.64-13.30)$ & $0.021^{\mathrm{a}}$ \\
\hline- & $9.23(3.68-30.94)$ & $7.05(4.62-22.97)$ & $0.017^{\mathrm{a}}$ \\
\hline \multicolumn{4}{|l|}{ Tumor size } \\
\hline$<2 \mathrm{~cm}$ & $10.1(5.80-26.54)$ & $7.98(3.64-22.97)$ & 0.061 \\
\hline$>2 \mathrm{~cm}$ & $8.97(3.68-31.61)$ & $6.81(4.30-12.52)$ & $0.007^{\mathrm{b}}$ \\
\hline \multicolumn{4}{|c|}{ Regional lymph node metastases } \\
\hline Present & $10.15(4.51-30.94)$ & $7.33(4.30-22.97)$ & $0.004^{\mathrm{b}}$ \\
\hline Absent & $9.05(3.68-31.61)$ & $7.52(3.64-14.68)$ & 0.059 \\
\hline \multicolumn{4}{|l|}{ Hormonal sensitivity } \\
\hline Hormonal-positive & $8.65(3.68-30.94)$ & $6.85(3.90-22.97)$ & $0.038^{\mathrm{a}}$ \\
\hline Hormonal-negative & $14.07(5.80-31.61)$ & $7.56(3.64-10.68)$ & $0.004^{\mathrm{b}}$ \\
\hline
\end{tabular}

${ }^{\mathrm{a}} \mathrm{P}<0.05,{ }^{\mathrm{b}} \mathrm{P}<0.01 .{ }^{\mathrm{c}} \mathrm{Median}$ (range).

(0.62-9.80 mmol/l) to $3.22 \mathrm{mmol} / \mathrm{l}$ (1.15-8.85 mmol/l) (Fig. 4). However, this increase was not significant $(\mathrm{P}=0.8984)$. There was no statistically significant change in TAC in any of the subgroups after 6-weeks of chemotherapy (Table VIII).

In the post-treatment period, neither APLN, RBP4 or 8-oxo-dG were shown to be correlated with the age of patients or their BMI. A positive correlation was observed between RBP4 and adipose tissue content, and between 8-oxo-dG and TAC values (Table IV).

\section{Discussion}

Breast cancer is the most common type of cancer in women worldwide, and is the second-leading cause of cancer-associated morbidity and mortality in women (44). The mechanisms and factors contributing to development and progression of breast cancer have been studied extensively. Adipocytokines and oxidative stress appear to serve a significant role in carcinogenesis $(45,46)$. Moreover, findings 
Table VIII. Effect of 6-weeks of chemotherapy on total antioxidant capacity concentration in women with breast cancer.

\begin{tabular}{lccr}
\hline Clinicopathological feature & Before chemotherapy, mmol/l & After 6-weeks of chemotherapy, mmol/l & P-value \\
\hline Histopathological grade & $2.98(1.26-12.13)$ & $3.2(1.15-8.85)$ & 0.626 \\
$\quad$ I/II & $2.91(0.62-9.20)$ & $3.66(1.42-8.72)$ & 0.481 \\
III & $3.57(0.62-12.13)$ & $3.5(1.42-7.47)$ & 0.684 \\
HER-2/neu expression & $2.91(1.26-9.20)$ & $3.22(1.15-8.85)$ & 0.700 \\
+ & & & \\
- & $2.85(0.62-12.13)$ & $3.5(1.15-8.72)$ & 0.284 \\
Tumor size & $3.32(2.02-9.20)$ & $3.22(1.52-8.85)$ & 0.316 \\
$<2 \mathrm{~cm}$ & & & 0.889 \\
$>2 \mathrm{~cm}$ & $3.48(1.26-12.13)$ & $3.27(1.52-8.85)$ & 0.983 \\
Regional lymph node metastases & $2.72(0.62-9.80)$ & $3.19(1.15-8.72)$ & 0.627 \\
Present & & $3.39(1.15-8.72)$ & 0.313 \\
Absent & $3.18(0.62-12.13)$ & $2.54(1.59-8.85)$ & \\
Hormonal sensitivity & $2.07(1.26-5.99)$ & & \\
Hormonal-positive & & & \\
Hormonal-negative &
\end{tabular}

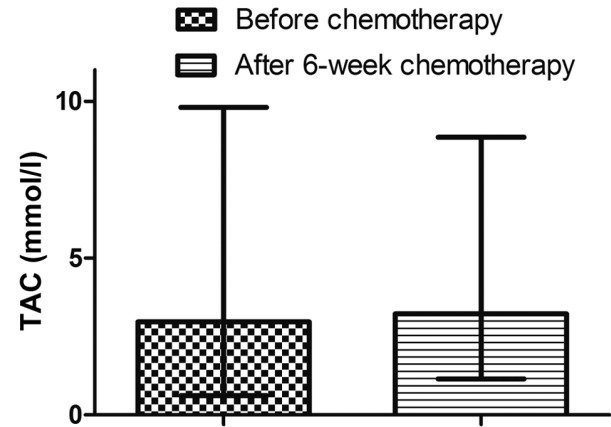

Figure 4. TAC values before and after 6-weeks of chemotherapy. Data were analyzed using a Wilcoxon test. TAC, total antioxidant capacity.

from several studies have suggested that adipocytokines and markers of oxidative stress may be promising tools for identifying advanced stage caner, lymph node metastases and adverse prognoses among cancer patients, including breast cancer patients $(47,48)$. Evaluation of these markers to predict the efficiency of anti-cancer treatments and survival outcomes in breast cancer patients is now becoming a subject of intense study $(49,50)$.

The aim of the present study was to evaluate the concentrations of APLN, RBP4 and 8-oxo-dG, as well as the TAC values, taking into account select clinicopathological characteristics of breast cancer, such as tumor histological grade and size, HER-2/neu expression, hormone receptor status, and the presence/absence of regional lymph node metastases. The evaluations were performed twice: before and after the second cycle of chemotherapy administration.

Increased APLN levels were found to be a significant and independent predictor of HER-2/neu expression. Women with a higher APLN concentrations appeared to be more likely to develop a positive HER-2/neu breast cancer phenotype. In general, HER-2/neu was overexpressed in $\sim 30 \%$ of breast carcinomas, and was associated with aggressive tumor behavior and a poor prognosis. Interestingly, HER-2 expression and functions have been shown to be modified by obesity and/or lipid-related components (51). A growing number of studies indicate the ability of adipocyte-secreted factors, namely leptin, to activate signaling pathways involved in the upregulation of HER-2/neu expression (52-54). An independent association between APLN and positive HER-2/neu status was demonstrated for the first time in the present study, and this may suggest the involvement of APLN in the development of this breast cancer subtype. The association between APLN and HER-2/neu expression should thus be investigated further.

In contrast, RBP4 levels were not associated with any clinicopathological features of breast cancer in the pre-treatment period. Its concentration was found to be similar amongst patients regardless of tumor size and histological grade, HER-2/neu status, hormone receptor status and the presence/absence of regional lymph node metastases. A limited number of studies have investigated the association between adipocytokine levels and clinicopathological features of breast cancer, with differing results (55). Significant differences in the serum concentrations of adipocytokines with regard to histological subtype, clinical stage and metastasis status were reported in some studies, but not others (56). Earlier observations supported by the present study suggest that secretion patterns of adipocytokines are specific to individuals, and are strictly dependent on the type of cancer and its characteristic features. Certain adipocytokines may rise or fall based on the particular type or subtype of cancer, whereas others may stay constant. Disease progression may also affect their levels differently. Therefore, the use of multiple adipocytokines in a panel may be more informative and allow for better prognostic prediction.

In the present study, no association was found between increased breast tumor aggressiveness and invasiveness with increased 8-oxo-dG concentrations or reduced antioxidant status. 
Similar findings were reported by Himmetoglu et al (48), who found that 8-oxo-dG levels did not change between breast cancer patients divided into different groups based on tumor grade, tumor stage and the presence/absence of metastases. In addition, Zowczak-Drabarczyk et al (57) demonstrated that the mean TAC levels did not differ significantly in relation to lymph node or HER-2/neu expression status in patients with newly diagnosed breast cancer. These results may suggest that 8 -oxo-dG and TAC values are not useful for subtyping breast cancer and assessing its aggressiveness or metastatic invasiveness.

It is well established that obesity significantly increases the risk of breast cancer and is associated with increased tumor burden, histopathological grade and a higher incidence of lymph node metastasis (58). The mechanisms by which obesity contributes to breast cancer are complex and have not yet been fully elucidated. However, several reports have shown that adipocytokines may be major contributing factors to obesity-associated breast cancer (59). Their altered secretion patterns in obese adipose tissues may modulate tumor cell behavior, such as proliferation and migration $(45,55)$. In the present study, a significant association with adipose tissue content was observed only for RBP4. In contrast, APLN was not correlated with adipose tissue content nor with BMI. Previous reports comparing the levels of adipocytokines between obese and non-obese breast cancer patients have yielded inconsistent results (55). Some findings indicate that obese breast cancer patients (BMI $>25 \mathrm{~kg} / \mathrm{m}^{2}$ ) had lower adiponectin and higher leptin levels compared with non-obese patients $\left(\mathrm{BMI}<25 \mathrm{~kg} / \mathrm{m}^{2}\right.$ ) (60). However, concentrations of other major adipocytokines, such as resistin and visfatin were found to not be altered, regardless of BMI values (60). El-Benhawy et al (55) observed a higher level of vistafin in women with breast cancer compared with the control group. However, there was no significant difference in visfatin concentrations between obese and non-obese subjects in the same study group of patients (55). Moreover, visfatin concentration were not correlated with BMI (55). The authors hypothesized that there may be other significant sources of this adipocytokine other than adipose tissue. Lymphocytes, neutrophils and other immune system cells can secrete certain adipocytokines as inflammatory phase proteins based on the inflammatory status of breast cancer. Therefore, they are suspected to be an alternative source of adipocytokines. The lack of any relationship between APLN and BMI with adipose tissue content in the present study may suggest that other factors, which are not necessarily connected with adipose tissue, may influence its levels. Unexpectedly, there was a significant negative association between 8-oxo-dG levels and BMI values. Although previous cross-sectional studies have reported a similar correlation in certain types of cancer, the exact relationship between these two factors remains unclear $(61,62)$. Mizoue et al (62) postulated that weight loss induced by increased energy expenditure that accompanies certain types of cancer, leading to elevation of mitochondrial ROS production, may be reflected in the rise of 8-oxo-dG levels (63). Numerous studies have confirmed the relationship between the secretion of adipocytokines by adipose tissue, and the stimulation or inhibition of ROS production. Than et al (64) showed that APLN inhibits the production and release of ROS in adipocytes by increasing the expression of antioxidant enzymes such as catalase, superoxide dismutase 1 and glutathione peroxidase, and also inhibits the expression of enzymes with pro-oxidative properties. Conversely, Wang et al (65) demonstrated the effects of RBP4 on the stimulation of anion radical production by mitochondria in the aortic vascular endothelium. However, in the present study, a correlation between the concentrations of the selected adipocytokines and indicators of oxidative stress was not found.

Chemotherapy is the most frequently used treatment for breast cancer patients, contributing to the reduction in cause-specific mortality by lowering the risk of recurrence and metastasis (66). Responses to chemotherapy vary greatly amongst individuals, making it difficult to accurately predict the outcomes of the treatment. Chemotherapy affects biochemical processes and as a result, alters the levels of various molecules, including those detectable in the blood (67). Understanding the relationship between changes in the concentration profiles of multiple circulating markers following different chemotherapeutic regimens may help predict their effectiveness.

The present study is the first to evaluate the effects of adjuvant chemotherapy on circulating levels of APLN and RBP4 in women with breast cancer. Previously, Słomian et al (67) assessed the adipocytokine levels in patients with colorectal cancer who received palliative chemotherapy. They observed increased plasma levels of the anti-inflammatory protein adiponectin, and decreased plasma levels of visfatin and resistin. Coskun et al (68) performed a study in which they enrolled patients with breast cancer who underwent tumor resection and then received adjuvant chemotherapy and/or radiotherapy. The authors did not observe altered serum levels of visfatin, adiponectin or leptin in the patients. However, resistin levels were found to be increased. In the present study, there were no significant differences in levels of APLN and RBP4 regardless of the clinicopathological features of the tumor during administration of chemotherapy. The constant and unchanged concentrations of APLN and RBP4 were observed during chemotherapy likely exclude them as candidates for monitoring the effects of treatments in breast cancer patients.

The mechanism of action of certain chemotherapeutic agents have been definitively linked to the generation of free radicals which, in-turn, induce tumor-cell apoptosis (69). However, the majority of the drugs act in a non-specific manner, harming both malignant and normal cells to a similar degree. This is evident by the elevation of key markers of oxidative stress, and reduced plasma levels of antioxidants that are observed during chemotherapy (70). Free radicals produced during chemotherapy cause oxidative damage to important biomolecules including DNA. One of the most frequently studied markers of oxidative DNA damage is the production of 8-oxo-dG, when ROS reacts with guanine bases in DNA (71). Several studies have shown that oxidative stress levels, based on 8-oxo-dG levels in urine or blood serum, may be a useful biomarker for determining the response to radiotherapy and chemotherapy in cancer patients (33). In most cases, chemotherapy accompanied by ROS overproduction results in elevated levels of 8-oxo-dG in patients with various types of cancer $(72,73)$.

In contrast to previous reports, in the present study, decreased levels of 8-oxo-dG were observed during chemotherapy in almost all groups of breast cancer patients. One exception was women with tumor sizes $<2 \mathrm{~cm}$ and without lymph node metastases. Pour Khavari et al (33) investigated the serum levels of 8 -oxo-dG following chemotherapy in 
patients with upper gastrointestinal tumors, and found that a decrease in its concentration was associated with worse response to treatment and shorter progression-free survival. Thus, it is hypothesized that the low serum levels of 8-oxo-dG observed in the present study during chemotherapy may reflect enhanced systemic antioxidant defense in response to ROS production induced by chemotherapy. The increased production of antioxidants may assist in the prevention of ROS-induced DNA damage, leading to decreased formation of 8-oxo-dG. It has been suggested that this mechanism may also occur in tumor cells and lead to chemoresistance by offering these cells a growth advantage through evasion of apoptosis and necrosis caused by ROS (74). Thus, chemoresistance may explain why patients with the worst chemotherapy responses have lower serum levels of 8-oxo-dG (33).

Contrary to previous reports, which demonstrated depletion in antioxidant status caused by chemotherapy (70), in the present study, TAC in breast cancer patients remained stable or increased slightly. Similar results were found by Hewala and Abo Elsoud (75), who observed that chemotherapy in breast cancer patients had no significant effect on serum TAC values. Moreover, Subramanyam et al (76) demonstrated that chemotherapy resulted in a significant increase in blood serum TAC values in cervical cancer patients. These contrasting observations may be important evidence of adaptation of the antioxidant system to enhanced production of ROS induced by chemotherapy. A positive correlation between TAC value and rising 8-oxo-dG levels during chemotherapy observed in the present study further confirms this hypothesis.

The present study has several limitations. First, the number of participants was small. Due to the size of the group, no division into molecular subtypes of breast cancer was performed and no correlation between them and adipocytokines and markers of oxidative stress was investigated. An analysis of the correlation between such small groups of patients would give unreliable and possibly unrepresentative results. Therefore, these preliminary observations serve as a proof-of-concept and basis for future clinical investigations involving larger cohorts of patients. The study did not take into account the division into histological subtypes of breast cancer, as it may wrongly suggest that only women with a specific histological subtype of breast cancer participated in the present study. Factors that may influence the concentrations of the analyzed parameters, such as menopause status and smoking habit were not considered. Additionally, the effects of the complete chemotherapeutic regimen was not assessed, only the effects of the first phase on the concentration of adipocytokines and markers of oxidative stress were investigated. To confirm the clinical usefulness of 8-oxo-dG in women with breast cancer, a prospective cohort study is required to determine 8-oxo-dG levels after completion of chemotherapy to investigate its association with outcomes.

In conclusion, the present preliminary study demonstrated that high APLN levels were independently associated with HER/neu expression, and may therefore be useful in subtyping this aggressive type of breast cancer. Moreover, 8-oxo-dG, which decreased during chemotherapy, may act as a serum marker for monitoring treatment effects. However, further studies are required to validate the clinical potential of these parameters. This includes confirming these results in a larger study group and considering important aspects such as other health status measures not included in the present study, as well as duration of chemotherapy, including after completion of the full regimen vs. analysis at different timepoints during the course of treatment.

\section{Acknowledgments}

Not applicable.

\section{Funding}

This work was supported by Poznan University of Medical Sciences (grant no. 502-01-22283-700-03102).

\section{Availability of data and materials}

The datasets used and/or analyzed during the present study are available from the corresponding author on reasonable request.

\section{Authors' contributions}

JG, JJB, MB, BG, MPK, WK, EL and MI conceived the study. $\mathrm{JG}, \mathrm{JJB}, \mathrm{EL}$ and WK collected the data. JG, MB, BG, and MPK analyzed the data. JG, JJB, EL and MI performed the experiments. JG and MB wrote the manuscript. MI reviewed and edited the manuscript. All authors read and approved the final manuscript. $\mathrm{JG}$ and MB confirm the authenticity of all the raw data.

\section{Ethics approval and consent to participate}

This study was performed in line with the principles of the Declaration of Helsinki. Approval was granted by the Ethics Committee of Poznan University of Medical Sciences (approval no. 1016/16).

\section{Patient consent for publication}

Informed consent was obtained from all individual participants included in the study.

\section{Competing interests}

The authors declare that they have no competing interests.

\section{References}

1. Gershuni VM, Ahima RS and Tchou J: Obesity and breast cancer: A complex relationship. Curr Surg Rep 4: 14, 2016.

2. Rigby AJ, Ray M and Basu TK: Biochemical status of vitamin $\mathrm{A}$ in patients with malignant and benign breast disease. J Clin Biochem Nutr 13: 53-61, 1992.

3. De Pergola G and Silvestris F: Obesity as a major risk factor for cancer. J Obes 2013: 291546, 2013.

4. Wu Q, Li B, Li Z, Li J, Sun S and Sun S: Cancer-associated adipocytes: Key players in breast cancer progression. J Hematol Oncol 12: 95, 2019.

5. Chu DT, Phuong TNT, Tien NLB, Tran DK, Nguyen TT, Thanh VV, Quang TL, Minh LB, Pham VH, Ngoc VTN, et al: The effects of adipocytes on the regulation of breast cancer in the tumor microenvironment: An update. Cells 8: 857, 2019.

6. Li J and Han X: Adipocytokines and breast cancer. Curr Probl Cancer 42: 208-214, 2018.

7. Hou WK, Xu YX, Yu T, Zhang L, Zhang WW, Fu CL, Sun Y, Wu Q and Chen L: Adipocytokines and breast cancer risk. Chin Med J (Engl) 120: 1592-1596, 2007. 
8. Christodoulatos GS, Spyrou N, Kadillari J, Psallida S and Dalamaga M: The role of in breast cancer: Current evidence and perspectives. Curr Obes Rep 8: 413-433, 2019.

9. Cabia B, Andrade S, Carreira MC, Casanueva FF and Crujeiras AB: A role for novel adipose tissue-secreted factors in obesity-related carcinogenesis. Obes Rev 17: 361-376, 2016

10. Luo Y, Yang C, Ye M, Jin C, Abbruzzese JL, Lee MH, Yeung SC and McKeehan WL: Deficiency of metabolic regulator FGFR4 delays breast cancer progression through systemic and microenvironmental metabolic alterations. Cancer Metab 1: 21, 2013

11. Jiao C, Cui L, Ma A, Li N and Si H: Elevated serum levels of retinol-binding protein 4 are associated with breast cancer risk: A case-control study. PLoS One 12: e0167498, 2016.

12. Salman T, Demir L, Varol U, Akyol M, Oflazoglu U, Yildiz Y, Taskaynatan H, Cengiz H, Guvendi G, Kucukzeybek Y, et al: Serum apelin levels and body composition changes in breast cancer patients treated with an aromatase inhibitor. J Buon 21 $1419-1424,2016$

13. Uribesalgo I, Hoffmann D, Zhang Y, Kavirayani A, Lazovic J, Berta J, Novatchkova M, Pai TP, Wimmer RA, László V, et al: Apelin inhibition prevents resistance and metastasis associated with anti-angiogenic therapy. EMBO Mol Med 11: e9266, 2019.

14. Tatemoto K, Hosoya M, Habata Y, Fujii R, Kakegawa T, Zou MX, Kawamata Y, Fukusumi S, Hinuma S, Kitada C, et al: Isolation and characterization of a novel endogenous peptide ligand for the human APJ receptor. Biochem Biophys Res Commun 251: 471-476, 1998.

15. Kleinz MJ and Davenport AP: Emerging roles of apelin in biology and medicine. Pharmacol Ther 107: 198-211, 2005

16. Mughal A and O'Rourke ST: Vasculareffects of apelin: Mechanisms and therapeutic potential. Pharmacol Ther 190: 139-147, 2018

17. Muto J, Shirabe K, Yoshizumi T, Ikegami T, Aishima S, Ishigami K, Yonemitsu Y, Ikeda T, Soejima Y and Maehara Y: The apelin-APJ system induces tumor arteriogenesis in hepatocellular carcinoma. Anticancer Res 34: 5313-5320, 2014.

18. Berta J, Hoda MA, Laszlo V, Rozsas A, Garay T, Torok S, Grusch M, Berger W, Paku S, Renyi-Vamos F, et al: Apelin promotes lymphangiogenesis and lymph node metastasis. Oncotarget 12: 4426-4437, 2014.

19. Yang Y, Lv SY, Ye W and Zhang L: Apelin/APJ system and cancer. Clin Chim Acta 457: 112-116, 2016.

20. Lacquaniti A, Altavilla G, Picone A, Donato V, Chirico V, Mondello, Aloisi C, Marabello G, Loddo S, Buemi A, et al: Apelin beyond kidney failure and hyponatremia: A useful biomarker for cancer disease progression evaluation. Clin Exp Med 15: 97-105, 2015

21. Feng M, Yao G, Yu H, Qing Y and Wang K: Tumor apelin, not serum apelin, is associated with the clinical features and prognosis of gastric cancer. BMC Cancer 16: 794, 2016.

22. Kotnik P, Fischer-Posovszky P and Wabitsch M: RBP4: A controversial adipokine. Eur J Endocrinol 165: 703-711, 2011.

23. Graham TE, Yang Q, Bluher M, Hammarstedt A, Ciaraldi TP Henry RR, Wason CJ, Oberbach A. Jansson PA, Smith U and Kahn BB: Retinol-binding protein 4 and insulin resistance in lean, obese, and diabetic subjects. N Engl J Med 354: 2552-2563, 2006.

24. Fei W, Chen L, Chen J, Shi Q, Zhang L, Liu S, Li L, Zheng L and $\mathrm{Hu}$ X: RBP4 and THBS2 are serum biomarkers for diagnosis of colorectal cancer. Oncotarget 54: 92254-92264, 2017.

25. Wang Y, Wong Y and Zhang Z: Adipokine RBP4 drives ovarian cancer cell migration. J Ovarian Res 11: 29, 2018.

26. Uzan J, Laas E, Alsamad IA, Skalli D, Mansouri D, Haddad B and Touboul C: Supervised clustering of adipokines and hormonal receptors predict prognosis in a population of obese women with type 1 endometrial cancer. Int J Mol Sci 18: 1055, 2017.

27. Kruk $\mathrm{J}$ and Aboul-Enein HY: Reactive oxygen and nitrogen species in carcinogenesis: Implications of oxidative stress on the progression and development of several cancer types. Mini Rev Med Chem 17: 904-919, 2017.

28. Waris G and Ahsan H: Reactive oxygen species: Role in the development of cancer and various chronic conditions. J Carcinog 5 : $14,2006$.

29. Ivanova D, Zhelev Z, Aoki I, Bakalova R and Higashi T: Overproduction of reactive oxygen species-obligatory or not for induction of apoptosis by anticancer drugs. Chin J Cancer Res 28: 383-396, 2016.

30. Berstein LM, Poroshina TE, Kovalenko IM and Vasilyev DA: Serum levels of 8-hydroxy-2'-deoxyguanosine DNA in patients with breast cancer and endometrial cancer with and without diabetes mellitus. Bull Exp Biol Med 161: 547-549, 2016.
31. Opanuraks J, Boonla C, Saelim C, Kittikiwit W, Sumpatanukul P, Honglertsakul $\mathrm{C}$ and Tosukhowong P: Elevated urinary total sialic acid and increased oxidative stress in patients with bladder cancer. Asian Biomed 4: 703-710, 2010.

32. Roszkowski K, Jozwicki W, Blaszczyk P, Mucha-Malecka A and Siomek A: Oxidative damage DNA: 8-oxoGua and 8-oxodG as molecular markers of cancer. Med Sci Monit 17: CR329-CR333, 2011.

33. Pour Khavari A, Liu Y, He E, Skog S and Haghdoost S: Serum 8-Oxo-dG as a predictor of sensitivity and outcome of radiotherapy and chemotherapy of upper gastrointestinal tumors. Oxid Med Cell Longev 2018: 4153574, 2018.

34. Di Meo S, Reed TT, Venditti P and Victor VM: Role of ROS and RNS sources in physiological and pathological conditions. Oxid Med Cell Longev 2016: 1245049, 2016.

35. Zowczak-Drabarczyk MM, Murawa D, Kaczmarek L, Połom K and Litwiniuk M: Total antioxidant status in plasma of breast cancer patients in relations to ER $\beta$ expression. Contemp Oncol (Pozn) 17: 499-503, 2013

36. Dludla PV, Nkambule BB, Jack B, Mkandla Z, Mutize T, Silvestri S, Orlando P, Tiano L, Louw J and Mazibuko-Mbeje SE: Inflammation and oxidative stress in an obese state and the protective effects of gallic acid. Nutrients 11: 23, 2018

37. Ren Y, Li Y, Yan J, Ma M, Zhou D, Xue Z, Zhang Z, Liu H, Yang H, Jia L, et al: Adiponectin modulates oxidative stress-induced mitophagy and protects $\mathrm{C} 2 \mathrm{C} 12$ myoblasts against apoptosis. Sci Rep 7: 3209, 2017.

38. Frühbeck G, Catalán V, Rodríguez A, Ramírez B, Becerril S, Salvador J, Portincasa P, Colina I and Gómez-Ambrosi J: Involvement of the leptin-adiponectin axis in inflammation and oxidative stress in the metabolic syndrome. Sci Rep 7: 6619, 2017.

39. Jakovcevic D, Dedic-Plavetic N, Vrbanec D, Jakovcevic A and Jakic-Razumovic J: Breast cancer molecular subtypes and oxidative DNA damage. Appl Immunohistochem Mol Morphol 23: 696-703, 2015.

40. Li JC, Yi F, Diao S and Li JY: Association between plasma adiponectin and risk of breast cancer by molecular subtypes. Sichuan Da Xue Xue Bao Yi Xue Ban 50: 708-713, 2019 (In Chinese).

41. Januškevičienė I and Petrikaitė V: Heterogeneity of breast cancer: The importance of interaction between different tumor cell populations. Life Sci 239: 117009, 2019.

42. World Medical Association: World Medical Association Declaration of Helsinki: Ethical Principles for Medical Research Involving Human Subjects. JAMA 310: 2191-2194, 2013

43. American Joint Committee on Cancer. AJCC Cancer Staging Manual. 7th edition. Springer, New York, NY, 2010.

44. Bray F, Ferlay J, Soerjomataram I, Siegel RL, Torre LA and Jemal A: Global cancer statistics 2018: GLOBOCAN estimates of incidence and mortality worldwide for 36 cancers in 185 countries. CA Cancer J Clin 68: 394-424, 2018.

45. Divella R, De Luca R, Abbate I, Naglieri E and Daniele A Obesity and cancer: The role of adipose tissue and adipocytokines-induced chronic inflammation. J Cancer 15: 2346-2359, 2016.

46. Klaunig JE and Kamendulis LM: The role of oxidative stress in carcinogenesis. Annu Rev Pharmacol Toxicol 44: 239-267, 2004.

47. Han C, Zhang HT, Du L, Liu X, Jing J, Zhao X, Yang X and Tian B: Serum levels of leptin, insulin, and lipids in relation to breast cancer in China. Endocrine 26: 19-24, 2005.

48. Himmetoglu S, Dincer Y, Ersoy YE, Bayraktar B, Celik V and Akcay T: DNA oxidation and antioxidant status in breast cancer. J Investig Med 57: 720-723, 2009.

49. De Rossi T, Panis C, Victorino VJ, Freitas LF, Herreral ACSA, Cecchini AL and Cecchini R: Breast cancer and oxidative stress in chemotherapy. Appl Cancer Res 29: 150-156, 2009.

50. Vera-Ramirez L, Sanchez-Rovira P, Ramirez-Tortosa MC, Ramirez-Tortosa CL, Granados-Principal S, Lorente JA and Quiles JL: Oxidative stress status in metastatic breast cancer patients receiving palliative chemotherapy and its impact on survival rates. Free Radic Res 46: 2-10, 2012.

51. Ray A: Tumor-linked HER2 expression: Association with obesity and lipid-related microenvironment. Horm Mol Biol Clin Investig: 32, 2017 doi: 10.1515/hmbci-2017-0020.

52. Giordano C, Vizza D, Panza S, Barone I, Bonofiglio D, Lanzino M, Sisci D, De Amicis F, Fuqua SA, Catalano S and Andò S: Leptin increases HER2 protein levels through a STAT3mediated up-regulation of Hsp90 in breast cancer cells. Mol Oncol 7: 379-391, 2013 
53. Soma D, Kitayama J, Yamashita H, Miyato H, Ishikawa $M$ and Nagawa H: Leptin augments proliferation of breast cancer cells via transactivation of HER2. J Surg Res 149: 9-14, 2008.

54. Fiorio E, Mercanti A, Terrasi M, Micciolo R, Remo A, Auriemma A, Molino A, Parolin V, Di Stefano B, Bonetti F, et al: Leptin/HER 2 crosstalk in breast cancer: In vitro study and preliminary in vivoanalysis. BMC Cancer 8: 305, 2008.

55. El-Benhawy SA, Abd El Moneim NA and Ebeid SA: Serum adipocytokines (Visfatin and Resistin): New biomarkers of breast carcinogenesis. Middle East J Cancer 6: 253-265, 2015.

56. Chen DC, Chung YF, Yeh YT, Chaung HC, Kuo FC, Fu OY, Chen HY, Hou MF and Yuan SSF: Serum adiponectin and leptin levels in Taiwanese breast cancer patients. Cancer Lett 237: 109-114, 2006.

57. Zowczak-Drabarczyk M, Murawa D, Połom K, Szarszewska M, Nowakowski W and Mańczak M: Plasma total antioxidant status in breast cancer women in relation to lymph node involvement and HER-2/neu expression. Rep Pract Oncol Radiother 12 319-322, 2007.

58. Picon-Ruiz M, Morata-Tarifa C, Valle-Goffin JJ, Friedman ER and Slingerland JM: Obesity and adverse breast cancer risk and outcome: Mechanistic insights and strategies for intervention. CA Cancer J Clin 6: 378-397, 2017.

59. Lee $\mathrm{CH}$, Woo YC, Wang Y, Yeung CY, Xu A and Lam KSL: Obesity, adipokines and cancer: An update. Clin Endocrinol 83 . 147-156, 2015.

60. Gui Y, Pan Q, Chen X, Xu S, Luo X and Chen L: The association between obesity related adipokines and risk of breast cancer: A meta-analysis. Oncotarget 8: 75389-75399, 2017.

61. Loft S, Vistisen K, Ewertz M, Tjønneland A, Overvad K and Poulsen HE: Oxidative DNA damage estimated by 8-hydroxydeoxyguanosine Excretion in humans: Influence of smoking, gender and body mass index. Carcinogenesis 13: 2241-2247, 1992.

62. Mizoue T, Kasai H, Kubo T and Tokunaga S: Leanness, smoking, and enhanced oxidative DNA damage. Cancer Epidemiol Biomarkers Prev 15: 582-585, 2006.

63. Mizoue T, Tokunaga S, Kasai H, Kawai K, Sato M and Kubo T: Body mass index and oxidative DNA damage: A longitudinal study. Cancer Sci 98: 1254-1258, 2007.

64. Than A, Zhang X, Leow MK, Poh CL, Chong SK and Chen P: Apelin attenuates oxidative stress in human adipocytes. J Biol Chem 289: 3763-3774, 2014.

65. Wang J, Chen H, Liu Y, Zhou W, Sun R and Xia M: Retinol binding protein 4 induces mitochondrial dysfunction and vascular oxidative damage. Atherosclerosis 240: 335-344, 2015.
66. Nies YH, Ali AM, Abdullah N, Islahudin F and Shah NM: A qualitative study among breast cancer patients on chemotherapy: Experiences and side-effects. Patient Prefer Adherence 12: 1955-1964, 2018

67. Słomian G, Świętochowska E, Nowak G, Pawlas K, Żelazko A and Nowak P: Chemotherapy and plasma adipokines level in patients with colorectal cancer. Postepy Hig Med Dosw 71: 281-290, 2017.

68. Coskun T, Kosova F, Ari Z, Sakarya A and Kaya Y: Effect of oncological treatment on serum adipocytokine levels in patients with stage II-III breast cancer. Mol Clin Oncol 4: 893-897, 2016.

69. Conklin KA: Chemotherapy-associated oxidative stress: Impact on chemotherapeutic effectiveness. Integr Cancer Ther 3: 294 2014.

70. Abdel-Salam OME, Youness ER and Hafez HF: The antioxidant status of plasma in patients with breast cancer undergoing chemotherapy. Open J Mol Integr Physiol 1: 29-35, 2011.

71. Chernikov AV, Gudkov SV, Usacheva AM and Bruskov VI: Exogenous 8-oxo-7,8-dihydro-2'-deoxyguanosine: Biomedical properties, mechanisms of action, and therapeutic potential. Biochemistry (Mosc) 82: 1686-1701, 2017.

72. Roszkowski K, Filipiak J, Wisniewska M, Mucha-Malecka A and Basta P: Potential survival markers in cancer patients undergoing chemotherapy. Clin Exp Med 15: 381-387, 2015.

73. Crohns M, Saarelainen S, Erhola M, Alho $\mathrm{H}$ and Kellokumpu-Lehtinen P: Impact of radiotherapy and chemotherapy on biomarkers of oxidative DNA damage in lung cancer patients. Clin Biochem 42: 1082-1090, 2009.

74. Sova H, Jukkola-Vuorinen A, Puistola U, Kauppila S and Karihtala P: 8-hydroxydeoxyguanosine: A new potential independent prognostic factor in breast cancer. Br J Cancer 102: 1018-1023, 2010.

75. Hewala TI and Abo Elsoud MR: The clinical significance of serum oxidative stress biomarkers in breast cancer females. Med Res J 4: 1-7, 2019.

76. Subramanyam D, Subbaiah KCV, Rajendra W and Lokanatha V: Serum selenium concentration and antioxidant activity in cervical cancer patients before and after treatment. Exp Oncol 35: 97-100, 2013.

This work is licensed under a Creative Commons Attribution-NonCommercial-NoDerivatives 4.0 International (CC BY-NC-ND 4.0) License. 